\title{
The Heat-Dissipation and Dehumidification System of the Hybrid Multi-Layer Gravity Heat Pipe Applied to Granary
}

\author{
Guoyong $\mathrm{Su}^{1}, \mathrm{Yu} \mathrm{Wu}^{1} \&$ Wei Gao ${ }^{1}$ \\ ${ }^{1}$ School of Mechanical Engineering, Anhui University of Science and Technology, Huainan, China \\ Correspondence: Guoyong Su, School of Mechanical Engineering, Anhui University of Science and Technology, \\ Huainan, 232001, China. E-mail: 451993958@qq.com
}

Received: May 15, 2019

doi:10.5539/mas.v13n8p76
Accepted: June 5, 2019

Online Published: July 27, 2019

URL: https://doi.org/10.5539/mas.v13n8p76

\begin{abstract}
Based on the basic working principle and heat transfer characteristics of gravity heat pipe in combination with the grain stack particle's stacking characteristics, this study changes the structure of traditional heat pipe to change the heat transfer mode between the grain stack and the gravity heat pipe so as to improve the grain's heat-dissipation rate and heat-dissipation efficiency. Generally, this system can satisfy the internal heat dissipation requirements of grain stack only under the action of a non-power fan driven by the air in the atmosphere and the temperature difference between inside and outside of the fan. When the internal temperature sensor of the grain stack detects that the internal temperature of the grain stack is high only under the action of the non-power fan, the pipeline booster fan will be started. At the same time, when the gas exchange occurs between the internal gas in grain stack and the external air, the dehumidification and drying of the grain stack can be realized through the gas drying device of the product. Through theory and simulation, this paper conducts a comparative analysis on the variation law of grain stack's temperatures under the action of gravity heat pipe and no gravity heat pipe so as to explore the heat-dissipation system's working efficiency of the new structure gravity heat pipe. The gravity heat pipe and the non-power fan in the system are all green products, which makes this design product have better heat-dissipation effect and less energy consumption.
\end{abstract}

Keywords: heat dissipation in grain stack, gravity heat pipe, non-power fan, green products

\section{Introduction}

\subsection{Current Situation of Modern Food Storage}

Currently, China's grain storage methods mainly include cold and low temperature storage, ventilation storage, gas storage, and chemical storage. Low temperature storage is relative to normal temperature storage. Generally speaking, it is at least 5 degrees lower than the temperature of the grain stored at normal temperature. Under the condition of not freezing and destroying stored grain, natural or mechanical refrigeration is applied to reduce the temperature of grain so as to inhibit the activity of various biological components in the grain stack, maintain the nutritional components, fresh taste and germination ability of stored grain, and alleviate aging (Yang G J, et al, 2012). The principle of controlled atmosphere storage is to change the composition of the gas in the storage environment, whose main technical approach is to inject carbon dioxide, nitrogen, or other organisms other than the stored materials in the airtight storage environment, consume oxygen in the grain stack, and increase the concentration of carbon dioxide and nitrogen so as to achieve insecticidal, inhibit mold, and effectively control the normal metabolism of stored goods (Yuan S H, 1994). Its largest advantage is that it can inhibit pests and diseases without the need of pesticides, which protects the safety of food use, and accords with the green development philosophy pursued by the contemporary. Therefore, China has invested heavily in the construction of a gas storage base, and achieved fruitful results. China's atmosphere storage technology is in a leading position in the world (Lan S B, et al, 2008). Chemical storage is a storage method to prevent and deal with many phenomena, such as mildew, insect formation, in the process of grain accumulation by using chemical agents. The improper use of chemical agents will cause great harm to human health. Therefore, China has identified many frequently-used chemical agents and methods, and developed many environmentally-friendly and non-toxic agents to replace agents harmful to the human body (Ma Y, 2014; Cui G H and Cao Y, 2004).

The realization of the above grain storage methods is based on the construction of modern factory buildings. However, on the one hand, the construction of modern factory buildings is very expensive, on the other hand, to 
maintain the temperature, humidity and other parameters in the grain warehouse, people need a large amount of pipeline booster fans. Moreover, it will consume a lot of electricity per day. The survey found that the construction of modern factories in township and village enterprises is rare. Most of the grain storage equipment is backward with poor grain storage conditions, so the quality of grain storage is difficult to be guaranteed.

\subsection{Application of Heat Pipe in Grain Storage Technology}

The heat pipe is a heat transfer element with high thermal conductivity developed in the 1960s, which can transmit a large heat load with a small temperature difference. In recent years, with the continuous maturity and deepening of research on heat pipe technology, heat pipes have been widely used in various fields such as construction, transportation, chemistry, textile, production and life. There are many types of heat pipes. The gravity type heat pipe is very suitable for deriving the heat inside the grain stack due to its small temperature difference, large heat transfer and isothermal (Shao S, et al, 2013; Shi J T, 2010; Li Z J, et al, 2014). At present, Cheng X L (2016) designed and invented an anti-gravity heat pipe for low-temperature grain storage, and applied the heat pipe to the rice silo to study and analyze the vertical and horizontal grain temperature of the rice silo in a single anti-gravity heat pipe. Based on heat pipe technology, Xiu F L (2013) designed and developed a storage granary using natural cold storage. Compared with ordinary storage granaries, the new storage granules with heat pipe technology have a faster cooling and a greater amount of cold storage. Han D (2009) used the gravity heat pipe to derive the heat emitted from the japonica rice stack, so as to insert the single and double gravity heat pipes into the japonica rice stack from the perspective of food safety, focusing on the effect of gravity heat pipe on the temperature distribution of japonica rice. The results show that the single heat pipe in the japonica rice stack has an influence on the temperature distribution within a radius of $75 \mathrm{~mm}$. And the presence of the heat pipe can significantly delay the temperature rise of the japonica rice stack. Therefore, applying the heat pipe to the granary can reduce the use intensity of the pipeline booster fan. Obviously, it has certain practical significance and potential market value. However, the application of this technology is still in the preliminary experimental stage. The use of heat pipes for heat dissipation theory of grain stack requires further research to improve heat dissipation.

\section{Description of Key Technical Issues Solved in Design and Production}

This design mainly solves the problem of local high temperature inside the grain stack and reduces the power consumption of the granary. Therefore, improving the heat dissipation efficiency of the heat pipe and reducing the power consumption are the key technology of this topic.

\subsection{Multi-Layer Gravity Heat Pipe Used in the Heat Dissipation Mechanism of Grain Reactor}

Gravity heat pipes mainly transmit heat through heat conduction and convection. However, the heat dissipation efficiencies of these two heat dissipation methods are quite different (Zhan L, et al, 2016; Wang S Q, et al, 2008; Li X Y, et al, 2015). Due to the special structure of the internal particles, the grain stack has a small amount of convective heat exchange between the gas in the gap and the heat pipe after being inserted into the heat pipe, and the heat is mainly carried away by heat conduction. However, the grain particles generally have a small thermal conductivity and a limited contact area with the heat pipe, so the heat transfer efficiency is greatly limited. In this paper, the heat transfer mechanism of gravity heat pipe with multi-layer structure is studied by changing the heat transfer mode between grain particles and heat pipe, so as to improve the heat transfer efficiency of gravity heat pipe.

\subsection{Heat Dissipation Mechanism of Hybrid Multi-Layer Gravity Heat Pipe}

There are many factors affecting the convective heat transfer coefficient, and the flow velocity of the fluid has a great influence on the heat transfer coefficient. Therefore, considering the actual situation of grain storage, it is easy to achieve the scheme of convective heat exchange effect between external equipment and heat pipe. This design used non-power fan and pipeline booster fan to provide hybrid power for gas flow around evaporation section of multi-layer gravity heat pipe. On the one hand, the gas flow rate directly affects the heat dissipation effect of grain stack, on the other hand, the reasonable distribution of the working state of two kinds of fans can realize the energy saving. Therefore, it is the focus of this research and design to study the heat dissipation effect of multi-layer gravity heat pipes under the combined action of two types of fans.

\section{Design Scheme}

\subsection{Working Principle}

The principle in the heat-dissipation and dehumidification system of the hybrid multi-layer gravity heat pipe is shown in figure 1. Under the action of air flow and internal and external temperature difference, the non-power fan rotates by itself. In the pipe connected to it, it will negative pressure, which will be transferred along the pipe 
to the inner and outer wall of the multi-layer gravity heat pipe. Under the action of negative pressure, the gas in the grain stack will flow to the multi-layer gravity heat pipe to exchange heat with it, and the temperature of the grain reactor will decrease. The temperature sensor monitors the temperature of each measuring point from time to time. When the temperature is higher than the safe temperature of the grain stack, the pipeline supercharging fan will be started automatically to increase the negative pressure between the multi-layer gravity heat pipes so as to promote the heat dissipation. Before the gas flows through the multi-layer heat pipe to the atmosphere in the grain stack, the gas drying device is applied to realize the dehumidification process in the grain stack. Its work flow is shown in figure 2 .

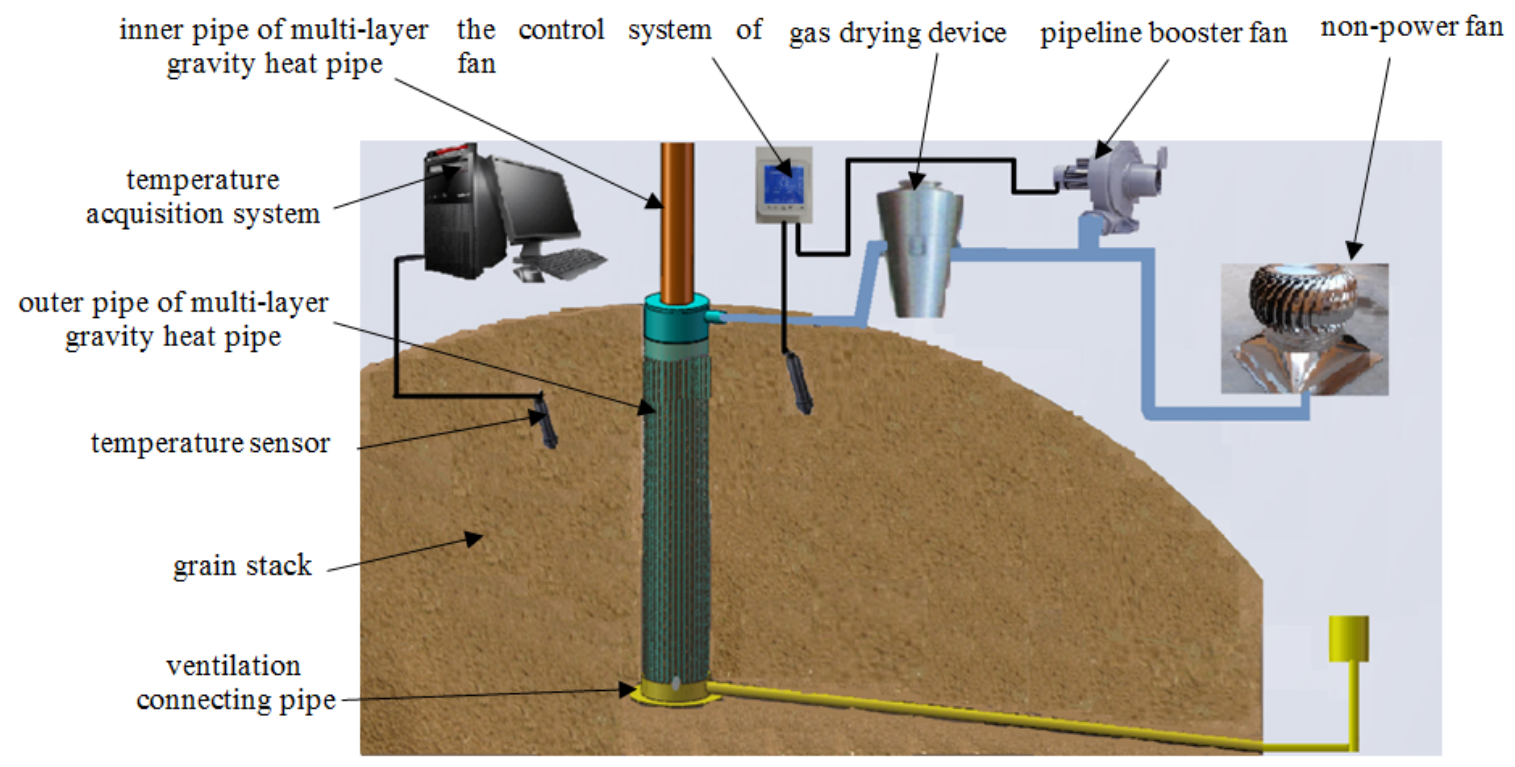

Figure 1. Experimental conception diagram of hybrid multi-layer gravity heat pipe heat-dissipating dehumidification system applied to granary

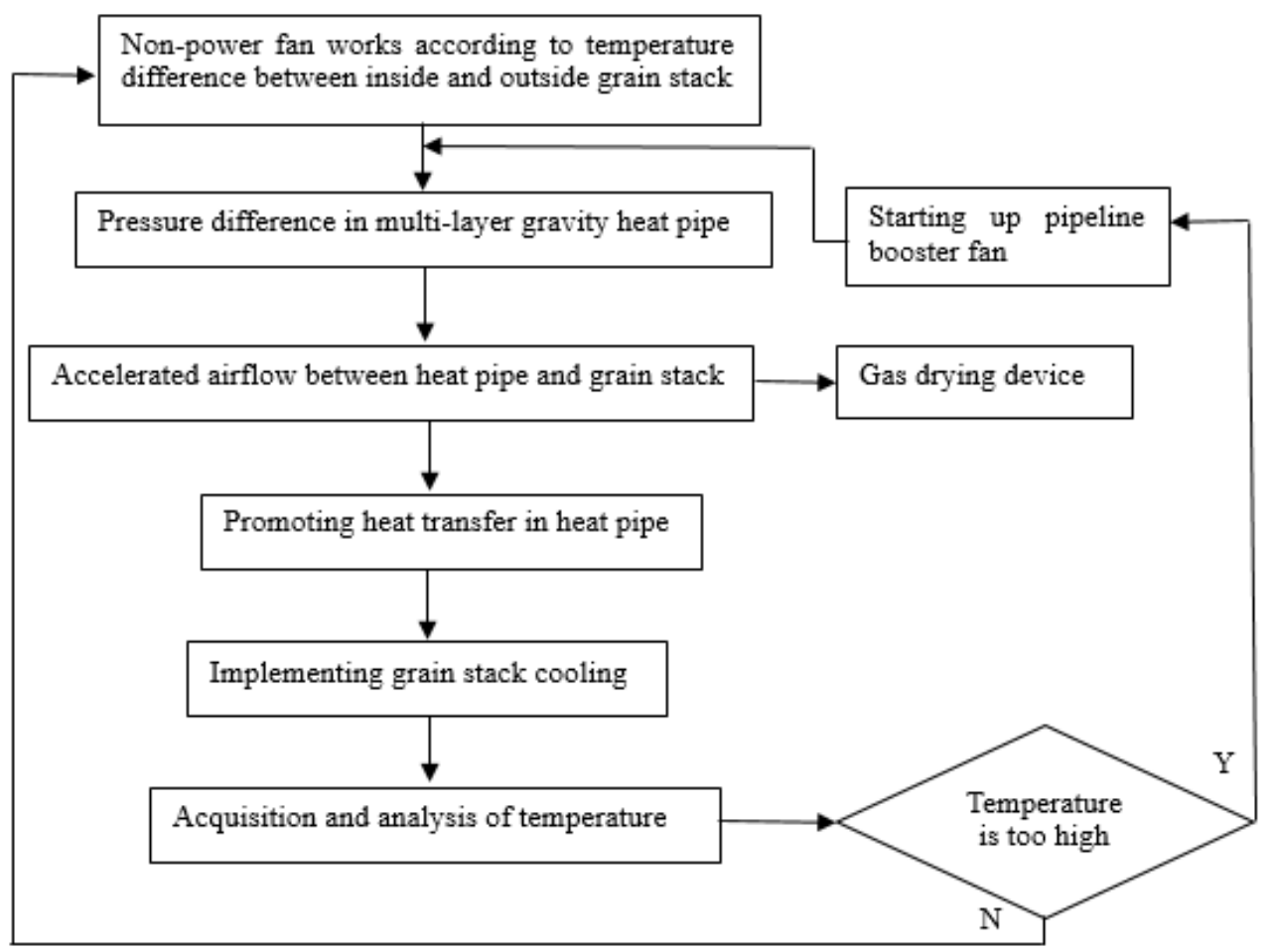

Figure 2. Working flow chart of hybrid multi-layer gravity heat pipe heat-dissipating dehumidification system applied to grain stack 


\subsection{Theoretical Model}

3.2.1 Mathematical Model of Heat Dissipation for Hybrid Multi-Layer Gravity Heat Pipe Heat -Dissipation and Dehumidification System

Because the multi-layer gravity heat pipe is placed in the grain heap, there is only heat transfer process between the grain heap and the heat pipe. Therefore, it is not necessary to consider the problem of gas phase mass transfer in the grain stack, and only the heat transfer is required to calculate the energy transfer. The heat transfer process of the multi-layer gravity heat pipe is shown in figure 3 . Where $t_{f}$ is the temperature in the rice stack; $t_{w l}$ is the temperature at the wall of the outer pipe of multi-layer gravity heat pipe; $t_{w 2}$ is the temperature of the outer wall of multi-layer gravity heat pipe's inner pipe; $t_{w 3}$ is the temperature at the inner wall of multi-layer gravity heat pipe's inner pipe; $t_{v}$ is the temperature of the liquid inside the gravity heat pipe; $D_{0}$ is The diameter of the multi-layer gravity heat pipe's outer pipe; $d_{i}$ and $d_{0}$, are the inner and outer diameters of the multi-layer gravity heat pipe's inner pipe; $t_{\infty}$ is the environment temperature.

Boundary condition:

$$
\tau>0, t_{f}=f(\tau)
$$

(1) Solid-phase energy conservation equation

$$
(1-\varepsilon) \rho_{s} c_{s} \frac{\partial t_{s}}{\partial \tau}=(1-\varepsilon) \lambda_{s}\left(\frac{\partial^{2} t_{s}}{\partial x^{2}}+\frac{\partial^{2} t_{s}}{\partial y^{2}}\right)
$$

Where:

$t_{s}$-The temperature of solid phase, $K$;

$\varepsilon$ - Porosity of porous medium in rice stack;

$\rho_{s}$-Density of solid phase, $\mathrm{kg} / \mathrm{m}^{3}$;

$c_{s}$ - The specific heat of solid phase, $\mathrm{kJ} / \mathrm{kg} \cdot \mathrm{K}$;

$\lambda$-Thermal conductivity of solid phase, $\mathrm{W} / \mathrm{m} \cdot \mathrm{K}$.

(2) Gas-phase energy conservation equation

$$
\varepsilon \rho_{g} c_{g} \frac{\partial t_{g}}{\partial \tau}=\varepsilon \lambda_{g}\left(\frac{\partial^{2} t_{g}}{\partial x^{2}}+\frac{\partial^{2} t_{g}}{\partial y^{2}}\right)
$$

Where:

$t_{g}$ - The temperature of gas phase, $K$;

$\rho_{g}$-Density of gas phase, $\mathrm{kg} / \mathrm{m}^{3}$;

$c_{g}$ —Specific heat of gas phase, $\mathrm{kJ} / \mathrm{kg} \cdot \mathrm{K}$;

$\lambda_{g}$-Thermal conductivity of gas phase, $W / m \cdot K$.

(3) Total Energy Equation

$$
\begin{gathered}
\rho c \frac{\partial t}{\partial \tau}=\lambda\left(\frac{\partial^{2} t}{\partial x^{2}}+\frac{\partial^{2} t}{\partial y^{2}}\right) \\
\lambda=(1-\varepsilon) \lambda_{s}+\varepsilon \lambda_{g} \\
\rho=(1-\varepsilon) \rho_{s}+\varepsilon \rho_{g} \\
c=(1-\varepsilon) c_{s}+\varepsilon c_{g}
\end{gathered}
$$

(4) Heat transferred by a multi-layer gravity heat pipe per unit time

$$
\begin{gathered}
Q=\frac{\Delta \mathrm{t}}{R} \\
R=R_{0}+R_{1}+R_{2}+R_{3}+R_{4}+R_{5}+R_{6}+R_{7}
\end{gathered}
$$

Where: 
$\rho$-Total density, $\mathrm{kg} / \mathrm{m}^{3}$;

$c$-Total specific heat, $\mathrm{kJ} / \mathrm{kg} \cdot \mathrm{K}$;

$\lambda$-Total thermal conductivity, $W / m \cdot K$;

$\Delta t$-Temperature difference between the interior of the grain stack and the environment, $K$;

$R_{0}$-The total thermal resistance between the grain stack particles and the outer pipe of multi-layer gravity heat pipe, $K / W$;

$R_{l}$-The thermal resistance from the outer pipe of multi-layer gravity heat pipe to the outer wall of the evaporation section, $K / W$;

$R_{2}$-The thermal resistance of the outer wall to the inner wall of the evaporation section, $K / W$;

$R_{3}$ - Heat transfer resistance of the evaporation section, $K / W$;

$R_{4}$-The thermal resistance caused by the pressure drop of the vapor flow from the evaporation section to the condensing section, $K / W$;

$R_{5}$ - Heat transfer resistance of condensation section, $K / W$;

$R_{6}$-Condensation section solid wall thermal resistance, $K / W$;

$R_{T}$-Heat transfer resistance between the outer wall of the condensation section and the atmosphere, $K / W$.

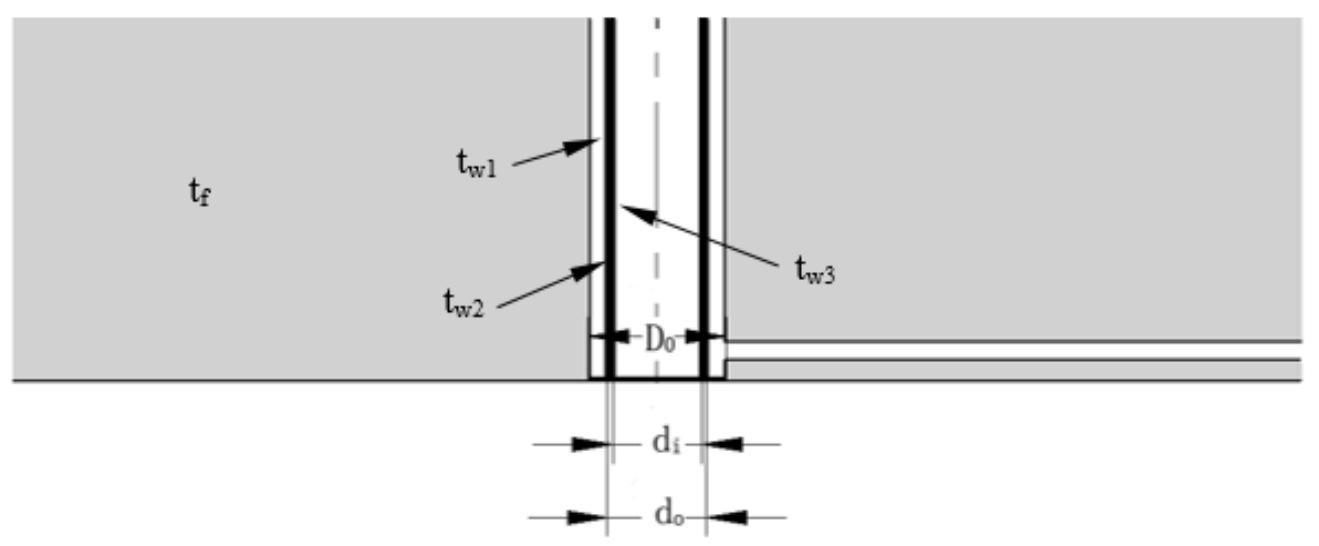

Figure 3. Heat transfer analysis of multi-layer gravity heat pipe

3.2.2 Mathematical model of heat dissipation in ordinary granary cooling system

The granary is dissipated as a fluid-extracting sphere by the heat dissipation of the grain stack under the action of natural ventilation.

$$
\begin{aligned}
N u=2+\left(0.4 \operatorname{Re}^{1 / 2}+0.06 \operatorname{Re}^{2 / 3}\right) \operatorname{Pr}^{0.4}\left(\frac{\eta_{\infty}}{\eta_{\mathrm{w}}}\right)^{1 / 4} \\
\mathrm{Re}=\frac{u l}{v} \\
\operatorname{Pr}=\frac{\mu c_{p}}{\lambda} \\
h=\frac{N u \lambda}{l} \\
R=\frac{2}{\pi D^{2} h}
\end{aligned}
$$




$$
Q=\frac{\Delta \mathrm{t}}{R}
$$

The heat and mass transfer equation of rice stack porous media with boundary conditions of the third kind:

Gas phase mass conservation equation:

Momentum conservation equation:

$$
\frac{\partial \rho_{g}}{\partial \tau}=-\left(\frac{\partial \rho_{g} u}{\partial x}+\frac{\partial \rho_{g} v}{\partial y}\right)
$$

$$
\begin{aligned}
& \rho_{g}\left(\frac{\partial u}{\partial \tau}+u \frac{\partial u}{\partial x}+v \frac{\partial u}{\partial y}\right)=F_{x}-\frac{\partial p}{\partial x}+\mu\left(\frac{\partial^{2} u}{\partial x^{2}}+\frac{\partial^{2} u}{\partial y^{2}}\right) \\
& \rho_{g}\left(\frac{\partial v}{\partial \tau}+u \frac{\partial v}{\partial x}+v \frac{\partial v}{\partial y}\right)=F_{y}-\frac{\partial p}{\partial x}+\mu\left(\frac{\partial^{2} v}{\partial x^{2}}+\frac{\partial^{2} v}{\partial y^{2}}\right)
\end{aligned}
$$

Energy conservation equation:

Where:

$$
(\rho c)_{g} \frac{\partial t}{\partial \tau}=\lambda\left(\frac{\partial^{2} t}{\partial x^{2}}+\frac{\partial^{2} t}{\partial y^{2}}\right)-\left(\frac{\partial \rho_{g} c_{g} u t}{\partial x}+\frac{\partial \rho_{g} c_{g} v t}{\partial y}\right)
$$

$D$-Diameter of the grain stack, $m$;

$v$-Dynamic viscosity of the gas phase, $\mathrm{m}^{2} / \mathrm{s}$;

$\eta_{0}$-Indoor Air Viscosity, Pa.s;

$\eta_{v}$-Air viscosity at the surface of the grain stack, $P a . s$;

$l$-Characteristic length, $m$;

$c_{g}$-Specific heat capacity of the gas phase, $\mathrm{kJ} / \mathrm{kg} \cdot \mathrm{K}$.

\subsection{Three-Dimensional Model Building}

After theoretical calculation, this study conducts the three-dimensional modeling on the mechanical structure of the heat-dissipation and dehumidification system of the hybrid multi-layer gravity heat pipe. The SolidWorks software is applied to design the structure of pipeline booster fan, ventilation duct, multi-layer gravity heat pipe, electric control cabinet, gas drying device inside the grain stack, arrangement of the heat-dissipation and dehumidification system and so on (see the model in figure 4). 


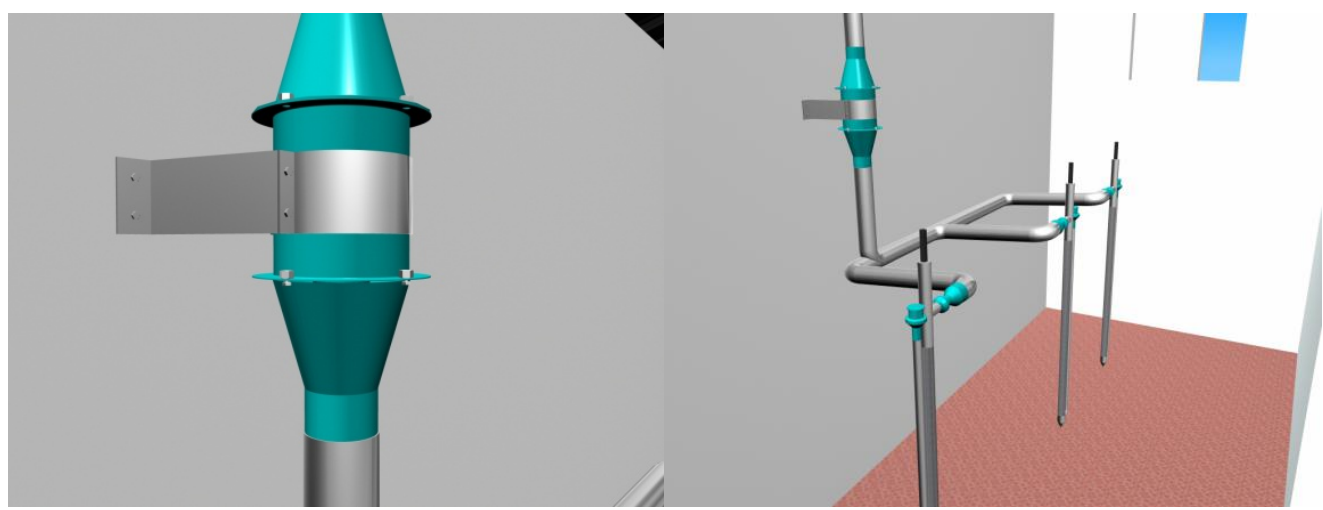

(a) Pipeline booster fan

(b) Arrangement of multi-layer gravity heat pipe

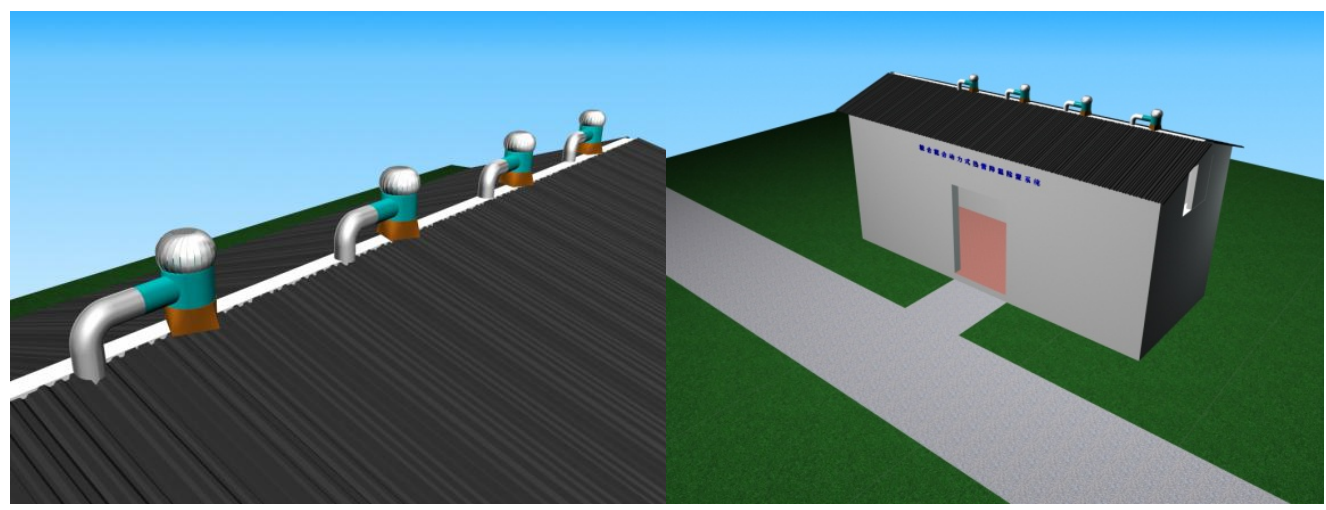

(c) Arrangement of non-power fan

(d) General effect diagram of heat dissipation in granary

Figure 4. Three-dimensional model of hybrid multi-layer gravity heat pipe heat-dissipation and dehumidification system applied in granary

\section{Simulation Analysis}

In order to study the heat dissipation mechanism of multi-layer gravity heat pipe applied in grain stack, the temperature distribution law of multi-layer gravity heat pipe is compared with that of common grain stack under the condition of long-term accumulation, and then the heat dissipation efficiency of multi-layer gravity heat pipe is analyzed.

Firstly, in the Gambit pretreatment software, an identical grain stack geometry model (the middle section is equivalent to a trapezoid of $3 \times 2 \times 1.55 \mathrm{~m}$ ) is established. In the grain stack model with the multi-layer gravity heat pipe, the size of heat pipe is set to $0.1 \mathrm{~m}$ in diameter and $1 \mathrm{~m}$ in height. And in mesh generation, elements are selected as quad/tri, type as pave, interval size 0.02 for meshing. The results of meshing are shown in figure 5 and figure 6.

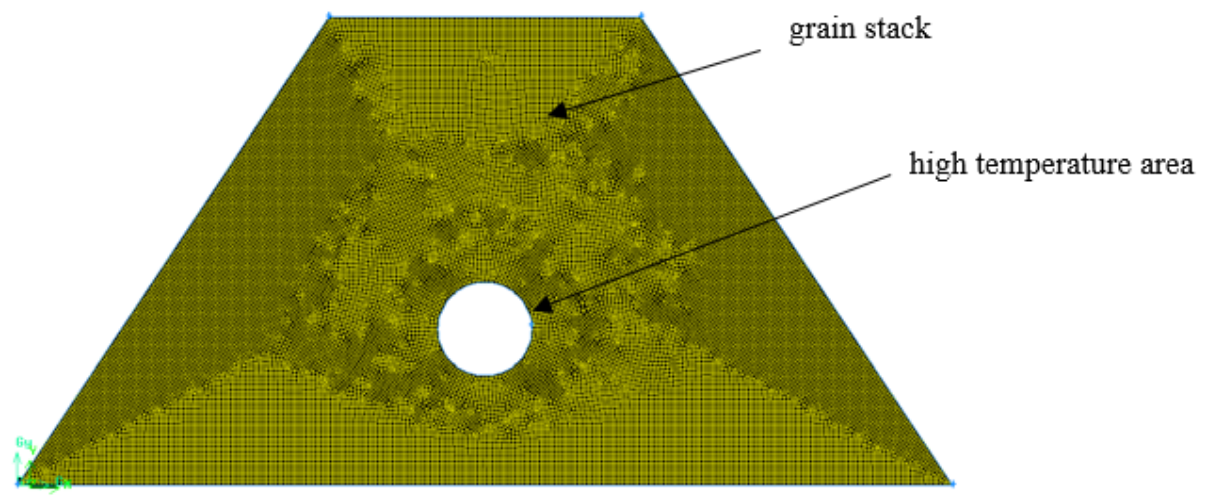

Figure 5. The grid graph of general grain stack simulation geometric model 


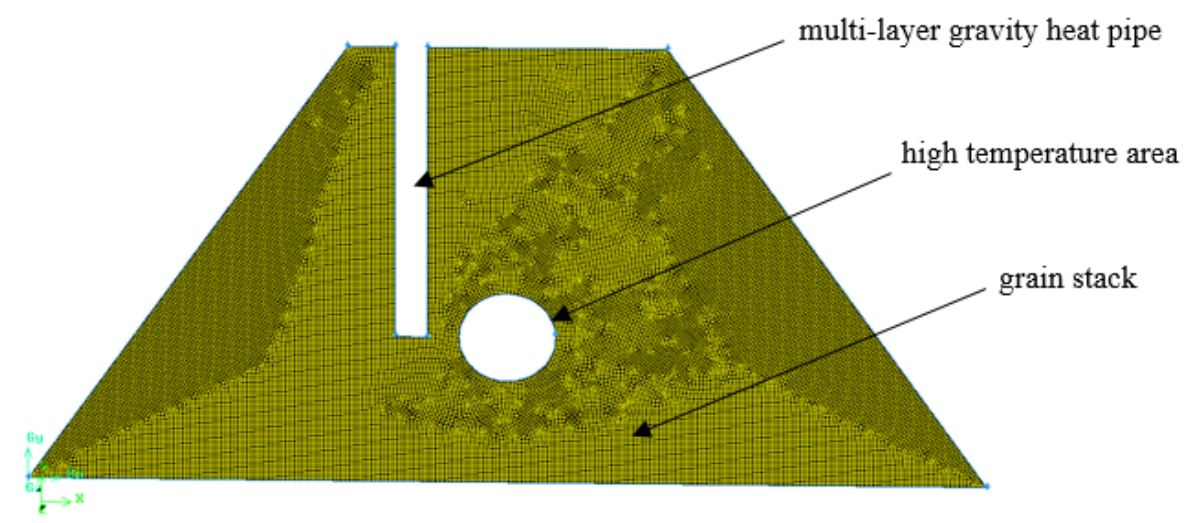

Figure 6. The grids of geometric model for grain stack simulation in this system

The above processed mesh model is imported into the ANSYS FLUENT software, and the boundary conditions, the property parameters of the grain stack, and the mathematical model of the simulation are set (the initial temperature of the grain stack is $291 \mathrm{~K}$ and the local high temperature zone inside the grain stack is assumed to be $303 \mathrm{~K}$. Rice as the simulation material, the porosity, the thermal conductivity and specific heat capacity of the rice stack are $0.629,0.0995 \mathrm{~W} / \mathrm{m} \cdot \mathrm{K}$ and $911.9 \mathrm{~J} / \mathrm{kg} \cdot \mathrm{K}$.). The simulation results are shown in figure 7, figure 8 , figure 9 , figure 10 .

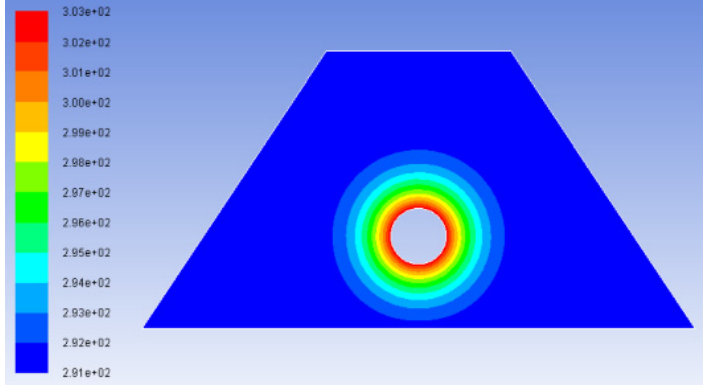

(a)

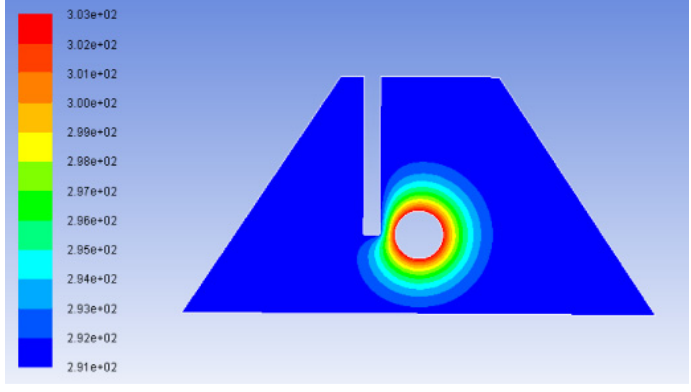

(b)

Figure 7.24-hour simulation results of grain stack

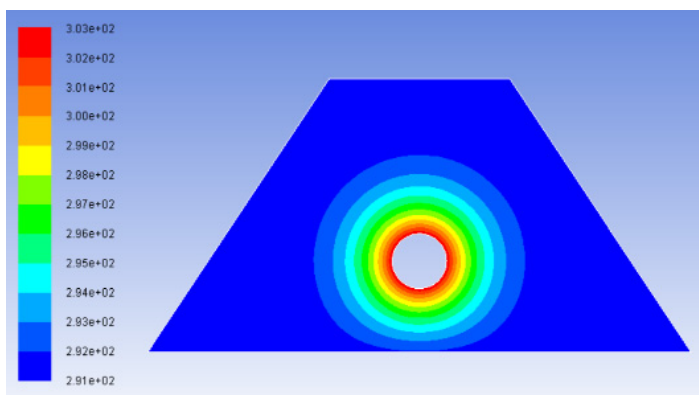

(a)

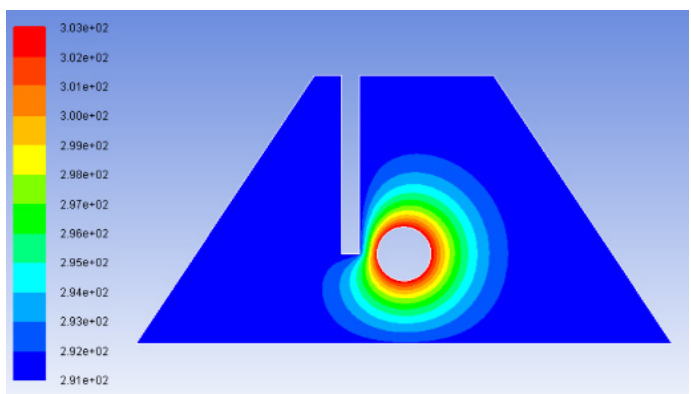

(b)

Figure 8. 48-hour simulation results of grain stack 


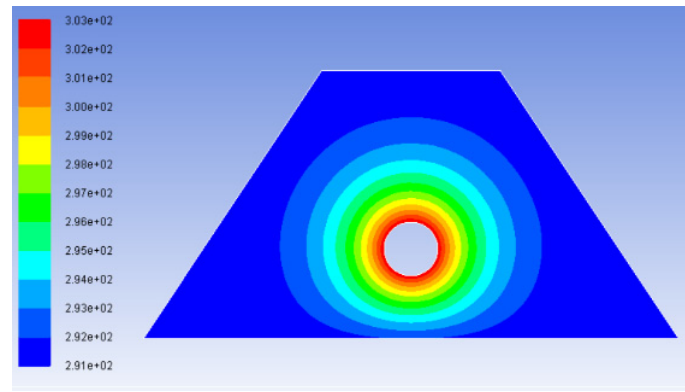

(a)

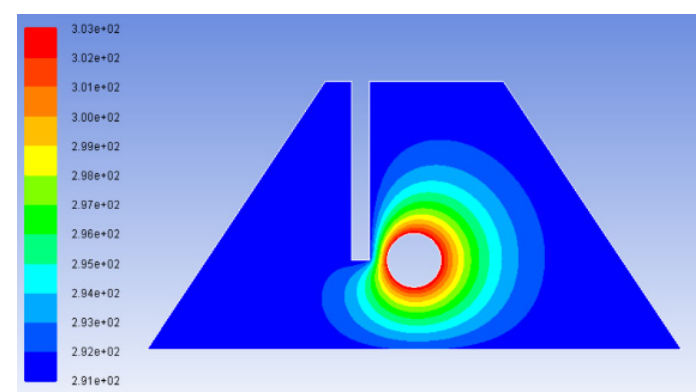

(b)

Figure 9. 96-hour simulation results of grain stack

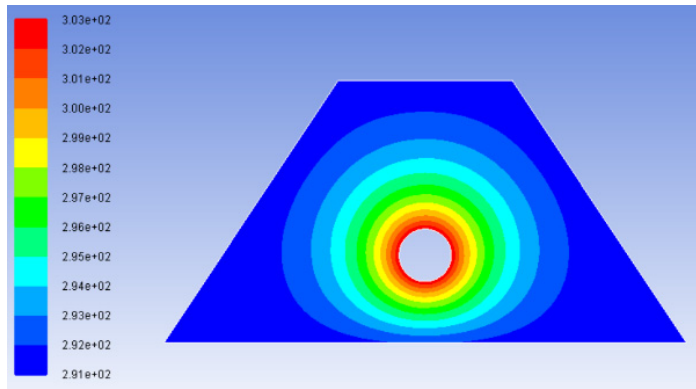

(a)

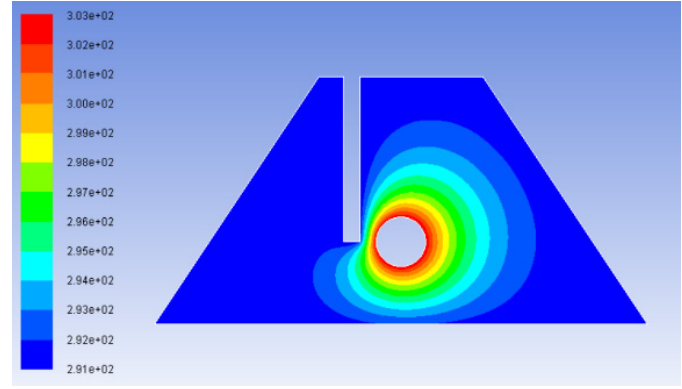

(b)

Figure 10. 144-hour simulation results of grain stack

As can be seen from figure 7, in the case of short stacking time, the effect of local high temperature on the surrounding temperature field is not obvious, and the heat dissipation of the grain stack is not obvious with or without multi-layer gravity. With the increase of grain stacking time (in figure 8 and figure 9), the temperature inside the grain stack will slowly affect the temperature around it. Even if the outer boundary of the grain stack is in the natural convection heat transfer, the cooling effect inside the grain stack is very limited, which cannot prevent its temperature from rising. Therefore, in order to ensure the quality of grain, it is particularly important to strengthen the heat transfer inside the grain stack. The distribution of temperature field in grain stack under the action of multi-layer gravity heat pipe is shown in figure 8 (b) and figure 9 (b). It can be seen that under the action of multi-layer gravity heat pipe, the effect of the internal high temperature zone of the grain stack on the surrounding grain temperature is lower than that of the ordinary grain stack during the same time of stacking. Especially in the area close to the multi-layer gravity heat pipe, the temperature drop is very obvious, which basically ensures that the grain temperature does not change significantly. From figure 10, we can see that the temperature field in the grain stack without multi-layer gravity heat pipe will continue to increase with the accumulation time, while in the grain stack with multi-layer gravity heat pipe, the temperature field in the grain stack will not increase with the extension of time. Therefore, it can be seen that the temperature of grain stack can quickly reduce under the action of the multi-layer heat pipe to meet the heat dissipation requirements.

\section{Conclusions}

1) The heat-dissipation and dehumidification system of the hybrid multi-layer gravity heat pipe was designed to improve the efficiency and rate of the heat dissipation.

2) The non-power fan is the main power source to generate negative pressure inside grain stack, and there is no need to consume electricity or other energy when working (Pipeline booster fan is auxiliary power equipment). Therefore, the system consumes less energy and is a green product.

3) By the academic analysis and the simulated result, it can be concluded that the new multi-layer gravity heat pipe has good heat dissipation effect. At the same time, this system is easy to install, which can be widely applied in warehouses and other places where temperature and humidity need to be maintained. 


\section{References}

Yang, G. J., Ren, Y. H. \& Jia, J. Y. (2012). The Current Situation and Development Trend of Grain Storage in China, Grain Processing, 01, 60-63.

Yuan, S. H. (1994). The Status Quo and Development Prospect of China's Grain Storage Technology. Journal of Nanjing University of Finance and Economics, 03, 49-53.

Lan, S. B., Guo, D. L. \& Yan, X. P. et al. (2008). Current Status and Future Development Trend of Grain Storage in China. Grain and Oil Storage Technology Newsletter, 04, 2-6. https://do i.org/ 10.3772/j.issn.1009-5659.2008.17.003

Ma, Y. (2014). Modern Granary and Green Grain Storage, Agricultural Science \&Technology, 01, 75-76. https://do i.org/10.3969/j.issn.1674-1161.2014.01.033.

Cui, G. H. \& Cao, Y. (2004). Application Practice and Development Suggestions of Low Temperature Storage of Grain, Grain Storage, (2), 20-24. https://do i.org/ 10.3969/j.issn.1000-6958.2004.02.005.

Shao, S., Feng, H. Q. \& Yang, X. X. et al. (2013). Analysis of Factors Affecting Pipe Wall Temperature of Radial Heat Pipe and Gravity Heat Pipe. Petro-chemical Equipment Technology, 02, 21-23 https://do i.org/ 10.3969/j.issn.1006-8805.2013.02.006.

Shi, J. T. (2010). Study on Heat Transfer Performance of Three-Phase Flow Gravity Heat Pipe, Tianjin: Tianjin University. https://do i.org/10.7666/d.y1925275

Li, Z. J., Wang, J. X. \& Wang, J. G. et al. (2014). Application of Heat Pipe Technology in Recovery of Waste Heat from Coal Mines, Energy and Energy Conservation, 04, 185-187. https://do i.org/ 10.3969/j.issn.2095-0802.2014.04.076.

Cheng, X. L. \& Gong, X. Z. (2016). Application of Anti-gravity Heat Pipe in Low Temperature Storage Grains, Grain Processing, 05, 48-50.

Xiu, F. L., Zhang, Y. \& Wang, S. Q. et al. (2013). Temperature Characteristics of Storage Silos Based on Heat Pipe Technology and Their Insecticidal Effects, Transactions of the Chinese Society of Agricultural Engineering, 14, 256-261. https://do i.org/ 10.3969/j.issn.1002-6819.2013.14.032.

Han, D., Peng, T. \& Li, Q. (2009). Experimental Study on The Influence of Gravity Heat Pipe on Temperature Distribution of Japonica Rice Stack, Journal of the Chinese Cereals and Oils Association, 04, 114-117.

Zhan, L., Sun, X. X. \& Lin, Z. G. et al. (2016). Discussion on the Factors Affecting Heat Pipe Heat Exchanger in Waste Heat Recovery of Boiler Exhaust Gas, Low Carbon-World, 29, 64-65.

Wang, S. Q. \& Song, Q. W. (2008). Preliminary study on technology for ice-making using natural cold resource based on heat pipe, Transactions of the Chinese Society of Agricultural Engineering, 24(11), 230-234. https://doi.org/10.3321/j.issn:1002-6819.2008.11.046.

Li, X. Y., Xiong, X. B. \& Zhang, Y. et al. (2015). Effect of Heat Pipe-Based Low-Temperature Grain Storage Technology on Wheat Quality, Journal of the Chinese Cereals and Oils Association, (1), 107-111. https://doi.org/ 10.3969/j.issn.1003-0174.2015.01.020.

\section{Copyrights}

Copyright for this article is retained by the author(s), with first publication rights granted to the journal.

This is an open-access article distributed under the terms and conditions of the Creative Commons Attribution license (http://creativecommons.org/licenses/by/4.0/) 